\title{
1. Introduction: a thing apart?
}

Adam Smith was in favour of pecuniary self-interest, rational choice and the invisible hand of supply and demand. The consumer and the shopper, he said, could be relied upon to produce the well-being of nations. The unique individual knows where the shoe pinches. The gain-seeking salesman knows whom he has to satisfy in order to live well: 'By pursuing his own interest he frequently promotes that of the society more effectually than when he really intends to promote it. I have never known much good done by those who affected to trade for the public good' (Smith, 1961 [1776]: I, 448).

Adam Smith looked to goal-orientated exchange to maximise people's welfare, self-perceived. Taken literally, his defence of devolution and factoring-down would suggest that health care is not a topic in public policy at all. The bodyholder in need of a recommendation or a procedure buys in the intervention of the specialist even as the butcher buys in beer from the brewer and the brewer buys in bread from the baker. The doctor, the nurse and the anaesthetist serve us not out of benevolence but with a view to their own profit, but at least they do serve us. Supply and demand are enough. Public policy is not needed. The State is not wanted. Shepherds and nannies need not apply.

This book takes a more pragmatic view. It argues that the free market is a good mechanism for allocating most goods and services but that the market for health has unusual properties that set it apart. This book explores the nature of those special characteristics. It argues that other commodities may have some of the unique properties but that health care is at the margins and the extremes in the extent to which it deviates from textbook supply and demand. The book asks if those properties mean that there is a role for social regulation and management. Apples and oranges can be left to the buyers and the sellers. But health care is different.

Chapter 2, Health Status and Health Care, says that health care is different because it is so difficult to define the inputs, list the outcomes or specify the production-function. All of human life is there. The inputs include not just the jabs and the plasters but the ban on cigarette advertising that paternalistically buffers the impressionable, the role-models who are defiantly not anorexic, alcoholic or on drugs, the subcultural peer pressures that encourage the couch potato to register for keep-fit. The outcomes are not just the sneeze arrested or the organ transplanted but the comfort of palliative care where there is no cure, 
the warmth of human contact where the doctor just listens to the friendless, the reintegration of the marginalised where the community nurse informs the housebound that there are welfare benefits for which they can apply. Health status is mortality and morbidity. Health care leads to amelioration and improvement. It is hard, however, to identify the stream that fills up the lake. It is even harder to know the precise relationship between the tool and the task that it is being expected to perform.

A needle and not a shovel is the appropriate instrument to sew on a button. People, valuing life and fearing death, are convinced that health care is itself the indispensable link in the chain that extends from symptom to cure. People have a strong resistance to pain, disease, depression, impairment of faculties, permanent disability, premature death. They believe that health care is the wonder drug that will turn the tables on misfortune and drive the Grim Reaper from the door. To that extent the doctrine is self-perpetuating. As we believe in God, so we believe in the medicine man, the shaman who can make miracles happen. The appeal is emotive and non-rational. No one likes to think that his life hangs by a shoestring or that there is no way to arrest his tremors.

Such wishful thinking is a fundamental psychological constant. Even though doctors themselves are uncertain and cannot therefore guarantee their results, nonetheless people follow the leader because the alternative is to have no lead at all. People demand health. Health is not for sale. Health care is the derived demand. It is the nearest the health-seeking can come to a nebulous core that no one knows how to make into an economic tradeable. Health care is different. It is different because people like to think that someone, somewhere knows what to do.

Chapter 3, Needs and Wants, says that health care is different because the consumer is ignorant, the future unknowable, and information asymmetry an invitation to abuse. The liberal economics of Smith, Mill and Pareto makes the assumption that the individual is the best judge of his own well-being. The argument for tolerance and patient sovereignty is called into question by the assertion that the shopper is not in a position to distinguish his wants from his needs or to protect himself against the supplier-induced demand of medical doctors with a for-profit goal-function of their own. Consumers are at the mercy of producers. Consumers do not know if they have an asymptomatic defect or what tests they require or where to go for good-quality service. They cannot decide if their demand curve is elastic or inelastic. They do not know what to do in unfamiliar territory where treatments are alternatives, markets are segmented, practitioners are differentiated and finance makes the world go round. Health care is instantaneous: the individual consumes the operation in the real time that the surgeon produces it. Health care is active: the patient must supply some input into making himself well. Health care, most of all, is unbalanced. The doctor knows best. Every patient knows that. 
The temptation to palm off a 'lemon' is a fact of life. Yet the doctor at the same time has a professional ethic which pulls him in the opposite direction. Asymmetrical information may in that sense be a greater threat when the consumer is buying a car or trying to decide if a chicken is fresh. The doctor's role as a carer makes him see himself as the agent of a purposive teleology that is more than crass money-making alone. Like an emergency clinic, he will not turn away a desperate client since his ultimate maximand is survival and health. $\mathrm{He}$ is often accredited to a non-profit supplier, either a private clinic or a British-type National Health Service. He works in highly regulated markets that circumscribe what he can and cannot do. Morality restricts flexibility and licensing filters entry. Competitive pricing, commercial advertising and entrepreneurial innovation can get a professional struck off. The butcher may have an instinct of workmanship but no one would say he had sworn a Hippocratic Oath that complements the law of contract. Health care, however, is different.

Society, meanwhile, has values and objectives of its own which it wishes to impose upon the dyad of market exchange. Health care involves other people. Other people want contagious externalities and third-party spillovers to be contained by collective action. As well as You and Me, there is also an Us which has a vision, a consensus and a baseline that is a common bond. Other people want absolute values like social justice, collective responsibility and respect for persons to complement the economist's maximand of input-output efficiency. Our common value system is what makes us fellow citizens, team-mates and integrated cooperators rather than isolated perfect competitors whose only purpose is to turn a penny whenever a gull is to be twisted. The market is about wants. Health care is about wants and about needs. Health care, in other words, is not the same.

Chapter 4, Payment for Health, says that health care is different because where people are insured it is other people who share the cost. Uncertainty is endemic: just as businesses do not know how fellow strategists will react and forecasters cannot be certain of the weather next Wimbledon, so consumers do not know what health care they will require, or when, or what it will cost. To reduce their vulnerability out-of-pocket to the unknown and the unanticipated, consumers often buy an entry ticket and share their risks as a club. There are no free riders where the baker sells bread to the butcher. All the costs and benefits are internalised in their swap. Insured health care, however, is exposed to a buffet syndrome. The socialism by consent is greater even than in other kinds of insurance. Fire or theft are unambiguous negativities that allow for little discretion or judgment. Health care, on the other hand, is an open-ended spectrum of alternative treatments and differing amenities. Not only can the diner eat as much as he likes, he can ask for different dishes, more expensive dishes to be served up. 
Where health insurance is a fringe benefit, it has spillover effects on employment, cost of production, economic growth and the mobility of labour. Where health insurance is subsidised by the nation, its integration into the tax/spend budgetary matrix makes it a public concern. Sometimes the subsidy is paid to the service: then the sense of citizenship is reinforced when black and white, rich and poor lie side by side in a common ward. Sometimes the subsidy is paid to the needy: then the deprived are means-tested up into a medical minimum and guaranteed a basic floor below which no human being will be allowed to sink. Sometimes the sponsorship is simply directed to the sick: all insurance is pooling and all pooling is cross-subsidisation. Nobody but a beggar expects such a hand-out when he walks into a bakery or a pub. Health care, however, is different.

Chapter 5, The Value of Life, continues the discussion of the patient, the practitioner and the public. It asks when enough is enough and the patient's life is no longer worth the cost. Millions spent on Jack is millions not spent on Jill. The nation cannot bury its head in the sand and pretend that a choice does not have to be made. The patient reveals his hidden trade-offs when he buys a cheap appliance that might give him an electrical shock or accepts danger-money to work at heights without a belt or harness. The practitioner gets involved when he says that clinical freedom means service to people and that professionals do not need the advice of dismal cost-effectiveness to tell them what to do about a life. Society takes a view when it says that it wants the limited supply of organs to be transplanted into productive high-flyers and not into retired has-beens who are no longer adding value for their tribe. Social expectations need not be more merciful than the market. For some thoroughly decent men they will be the ugly short-cut to the knacker's yard. The same must be said for some thoroughly decent women. This book uses 'he' and 'his' as a convenient shorthand to denote humankind as a whole. The language may be gendered but the argument is not.

Laissez-faire as a doctrine teaches that the butcher, the brewer and the baker should be trusted to buy and sell their own life-years. Where the patient is illinformed, however, where the agent advising his principal does not understand that his duty to Jack is at variance with his duty to Jill, there the market for a human life may be said to perform less well than when it is allocating meat to the highest bidder or beer to the thirsty shopper just willing to make the greatest sacrifice. Where, moreover, society is not satisfied with the market outcomes and wants to impose its own rankings on the passengers allowed first into the lifeboats, the market may be said to fail because it fails to satisfy the reasonable expectations of all the signatories to the social contract. The pressure of all upon each and the sui generis of mutual constraint has inexorably nationalised the scarce kidneys. The collectivity as a whole has said that it wants to make its voice heard. 
Chapter 6, Efficiency, says that health care is different because different doctors do different things and because randomised controlled trials seldom pick up the long-run consequences. That being the case, it is confusing to design a league-table of cost-effective treatments or to discount future earnings-streams saved over the expected life span of the representative bodyholder.

Health care is different as well because cost-effectiveness and cost-benefit analysis are seldom employed by the unique automaton. These techniques are more frequently used by an aggregate such as a nation to get value for money from a given endowment or to ensure that the internal rate of return matches society's next-best payoff from an identical outlay. Few individuals think of a hip transplant in terms of a three per cent payoff on human capital juxtaposed to an opportunity cost of five per cent from a Treasury bill. All governments, however, are expected to do so. Meat, beer and bread are not discounted by the nation when it decides whether to authorise the expense. But health care is different.

Chapter 7, Utility, is a reminder that cost-effectiveness and cost-benefit analysis are topics in economic psychology. Health care is desired not for its own sake but for the subjective satisfaction that the intervention will produce. That satisfaction in turn is both an end in itself (such as the pleasure of being able to walk unassisted) and a means to other ends (such as the self-respect that comes from being able to earn a living). It is difficult to know how to evaluate a status that is at once both an input and an outcome. No less is it difficult to gain a purchase on other people's invisible mind, or to add up all the images they are storing somewhere in their perception-base. Nor can the observer be confident that the evidence collected at great cost is free from irrational bias. Tradition, stereotyping, inertia, ideology, knee-jerk reflex are all psychological reasons why the probabilities might be distorted by the heuristic. So might an emotive response to the unknown. Risk-lovers enjoy taking a chance. Riskaverters feel that their hopes fall short of their fears. It is not easy to decide which member of a highly mixed community is the dead-centre representative who speaks with the voice of the whole.

Questionnaires try to find out the ordinal and the cardinal values that people put upon different procedures carrying different risks. Induction from revealed willingness to pay gives an insight into the intervals that separate the alternatives when people quantify their preferences through the money they spend. The bodyholder consulted, there is an argument that the practitioner and the public must be consulted as well if a robust measure of 'the good life' is to be constructed bottom-up by investigating the intuitions that most people most strongly hold. It would be bad economics to ask the butcher how much extra happiness he thinks the brewer obtains from one more slice of bread: interpersonal comparisons cannot reliably be made. In the area of health, however, such comparisons are, exceptionally, being made all the time. Health care is different. 
Chapter 8, Equity and Equality, considers the relationship between fairness and levelling. No one wants equal access to cinema-tickets, motorcars or pins. Access to doctors and nurses is, however, widely seen as a different breed of dog. Society feels strongly that it is only fair for all citizens to be guaranteed at least a basic package irrespective of their ability to pay. Perhaps this is because sickness and health, life and death are believed to be big issues that transcend the shopkeeper's calculus and the achievement-based reward. Health services are not the only basic goods (housing, education and food are also targeted for social support) but they are generally assigned a high ranking within the bundle. Health care is simultaneously a good thing in itself and the means to the attainment of other good things. Not everyone agrees on precisely what is just or on the frontier that separates the needs from the wants, the essential from the peripheral. Even so, there is near universal consensus that something should be done to level up the life-chances. The moral imperative casts a long shadow before.

Most of all does it do this in societies with religious values that preach compassion, altruism and solidarity, with historical traditions that are imbued with sharing, overlap and common culture. Even, however, in cultures that have invested heavily in meritocratic recognition and rising living standards, there is a surprisingly high level of commitment to equitable access and the infrastructure of health. T.H. Green on the State that levels up is alive and well in the clinics and the hospitals. Freedom is not just freedom from the State. It is the freedom to develop, to unfold, to become truly and fully oneself.

Chapter 9, Equality and Health, says that mortality and morbidity are not random but patterned. Disparities in outcomes are correlated with occupational hierarchy and geographical location. Low incomes make it more difficult for the deprived to pay for nutritious food, medical insurance and medical care. Subcultural mores mean that some strata are socialised into deferred gratification while others smoke heavily because they live for the day. Occupational differences are the reason why roofers have more falls from heights and lower-grade civil servants suffer more heart attacks. Medical attention, finally, is not available to all on the non-discriminatory basis of equal care for equal need.

The social failure suggests that there is scope for social intervention. Low incomes can be supplemented through housing allowances and child benefits. Subcultural conventions can be challenged through uninhibited mixing and health education. Occupational differences can be narrowed through works clinics and health and safety legislation. Medical care can be brought within each citizen's reach through community rating, non-exclusionary contracts, a National Health Service - and economic growth itself which makes all of us better able to afford the good things in life.

Chapter 10, Cost, issues a reminder that the cost of care is rising, both per unit of care consumed and as a share of the national product. A richer society 
spends more on meals out and luxury kitchens than does a poorer one. But health care is different. Hospitals in many areas are the regional multiplier that creates health-related jobs and keeps local people in work. Medical services in rust-belt countries are the sunrise sector that will make the nation an exporter and a hub. Producers as well as consumers have a vested interest in the health of health. The consequence is escalation. One dollar in every seven spent in the United States is being spent on formal care. This is more than in virtually any other nation. It was one dollar in every twenty in 1960 . What is being spent on the doctors is not being spent on the warships. Crowding out is on the horizon.

Chapter 11, Containing the Cost, concludes the book by suggesting public policies that can limit cost while not threatening health. Third-party payment, rising expectations, new technology, an ageing population all have contributed to a sector-specific rise that is well in excess of the rate of general inflation. So have supply-side imperfections such as professional bodies, local monopolies, the entry barrier of protracted training, the high capital cost of opening a new hospital, State intervention which limits the treatment centres in an attempt to keep down the cost of excess capacity, State subsidies which channel the treatments into areas that the market would have ignored. The market for care is not as competitive as the textbook ideal; but there are solutions which can be found to bend back the bent rod. The free market way forward would be to encourage entry and rivalry while doing away with counterproductive restrictions. The political way forward would be a greater reliance on charges, pecuniary incentives, waiting times, utilisation review to countervail the supplier's power to exploit, nationalisation of supply to reduce wasteful duplication, allocation by social criteria rather than by payment-led priorities lest effective demand starve the social organism of higher-valued utilities. Meat, beer and bread do not present so great a challenge as to necessitate a collective and a coordinated response. But health care is different. It is a thing apart. 